\title{
Targeting Cystic Fibrosis: A review from Pathogenesis to Innovative Clinical Strategies
}

\author{
Yiran $\operatorname{Liu}^{1 *}$ \\ ${ }^{1}$ Woodward Academy, College Park, Georgia, 30337, the United State;
}

\begin{abstract}
Cystic fibrosis is a hereditary disease caused by mutation in cystic fibrosis transmembrane conductance regulator (CFTR) gene, which controls CFTR proteins. Many are or have been threatened by this rare but life-threating disease, therefore, more attention should be paid to better solve this public health issue. Here we provide a thorough review about cystic fibrosis in the aspects of disease pathogenesis, clinical manifestation as well as clinical therapeutics to achieve a deeper understanding of this disease. Among the demand of innovative clinical strategies, appearance of the combination drug trikafta is a promising sign of better dealing with cystic fibrosis.
\end{abstract}

\section{Introduction}

Respiration is an essential activity to maintain vital signs. However, various respiratory diseases still persecute a large population in their health and daily lives. Cystic fibrosis (CF) is a type of uncommon hereditary diseases. In particular, patients inherit two copies from their parents. There is a complex chronic disease that affects more than 70,000 patients around the world. According to U.S. National Library of Medicine, cystic fibrosis affects about 1 in 2,500 to 3,500 white newborns, 1 in 17,000 African Americans, and 1 in 31,000 Asian Americans. Although it seems to be a rare disease, which there are over 70,000 patients around the world, it can be life-threatening. In old days, when medical technology was poor, many children with CF died before they reached school age. Improvement of medical care such as airway mucus clearance and controlling lung infection has greatly changed the epidemiology and demographics performances of cystic fibrosis [1, 2]. This disease is currently predominant in adult instead of children [3]. The number of adults with cystic fibrosis will continue to increase with almost all deaths occurring in this population [4]. Considering the growing threat of public health caused by cystic fibrosis, these is urgent attention requested worldwide. Here we present a thorough review about cystic fibrosis in the aspects of disease pathogenesis, clinical manifestation as well as clinical therapeutics.

\section{Pathogenesis of Cystic Fibrosis}

Cystic fibrosis is caused from gene mutation. Comprehensive description summarized from currently known mechanism [5-8] of the coding gene dysfunction is presented in Figure 1. Specifically, the underlying gene encodes a chloride-conducting transmembrane channel called the cystic fibrosis transmembrane conductance regulator (CFTR) and regulates anion transport and mucociliary clearance in the airways. Chloride ion outside of cells are essential for cilia -microscopic hair-like motile structure that removes dirt and mucus out of respiratory tract and helps people to breath normally. Chloride ions keep mucus and dirt in the airways to be moisture, thus celia could discharge them out of the airways simply. Normally, CFTR acts as chloride channel that pumps chloride from the intracellular space to the extracellular space thus facilitates casual respiratory [9]. Resulted from the disorder of CFTR protein, cells are unable to finish the chloride conductance process. In other words, lack of chloride ions causes difficult to normal ejectment of mucus and dirt, which may contain pathogenic bacteria or virus. Chunks of mucus would lead to problems to breathe. In additional, the dysfunction of CFTR also disrupts the regular proportion of sodium and water in epithelial sodium channel. Consequently, the mucus outside of the cell would have a greater viscosity. Both of these imbalances prompt mucus to stuck in lungs, then generate difficulties in breath and initiate cystic fibrosis. There are six classes of CFTR mutations in cystic fibrosis. Class I mutations produce no functional CFTR protein. Class II mutations, including the most prevalent type Phe508del (F508del)[10], cause retention of a misfolded protein at the endoplasmic reticulum, thus leading to degradation in the proteasome. Class III mutations affect channel regulation and impair channel opening. Class IV mutants show reduced conduction and decreased level of ions flow. Class V mutations cause substantial reduction in mRNA or protein, or both. Class VI mutations cause substantial plasma membrane instability. 


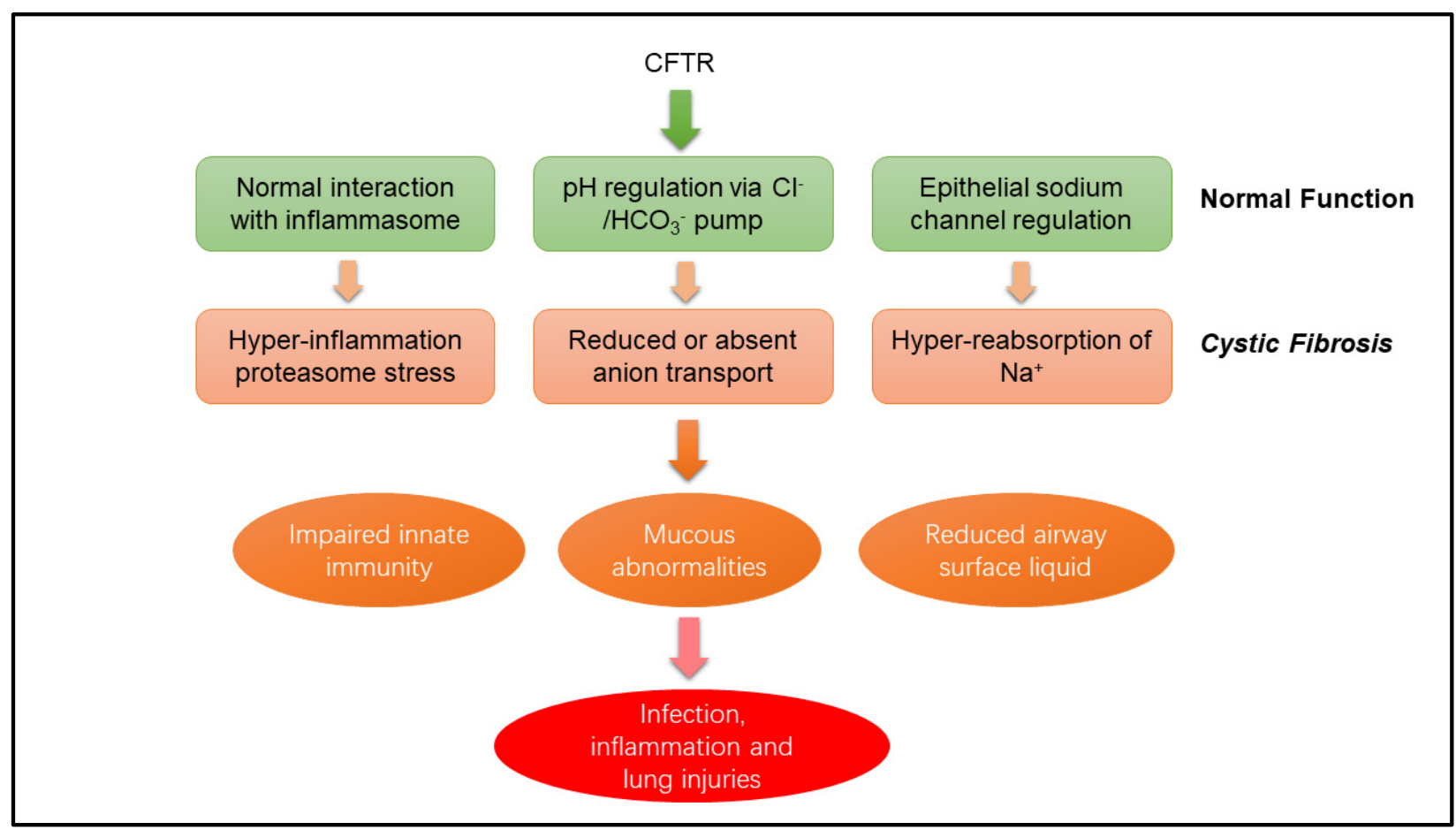

Figure1. CFTR dysfunction impact [11]

\section{Cystic Fibrosis Clinical Manifestation}

Originated from its pathogenesis, cystic fibrosis affects many body systems in addition to lung. As a hereditary disease, there are obvious phenotypic reference in different age groups (Table 2). The most significant manifestation is observed in respiratory system. Due to the pathology of CF, patients' breathing is generally unobstructed, often accompanied by cough, phlegm, infection, and inflammation. Bacteria that are embedded in mucus can induce pneumonia. Cystic fibrosis also has gastrointestinal symptoms. For patients in younger groups, exocrine pancreatic insufficiency can be generated by cystic fibrosis. What's more, over $80 \%$ of this kind of patients are accompany by exocrine pancreatic dysfunction[12].

Table 1. Onset of cystic fibrosis in approximate age group [11]

\begin{tabular}{|c|c|c|c|c|}
\hline & $0-10$ years & $10-20$ years & $20-35$ years & $>35$ years \\
\hline Airways & $\begin{array}{l}\text { Early mucinous plugging and } \\
\text { bronchiectasis }\end{array}$ & $\begin{array}{l}\text { Established } \\
\text { bronchiectasis }\end{array}$ & $\begin{array}{l}\text { Established bronchiectasis with } \\
\text { haemoptysis/pneumothorax }\end{array}$ & $\begin{array}{l}\text { Progressive respiratory } \\
\text { failure/lung transplant }\end{array}$ \\
\hline $\begin{array}{l}\text { Predominant } \\
\text { infection } \\
\text { source }\end{array}$ & Staphylococcus aureus & $\begin{array}{l}\text { S aureus/intermittent } \\
\text { Pseudomonas aeruginosa }\end{array}$ & $\begin{array}{l}\text { non-fermenting } \\
\text { Gram-negative bacteria } \\
\text { ( } P \text { aeruginosa), } \\
\text { allergic bronchopulmonary } \\
\text { aspergillosis }\end{array}$ & .. \\
\hline Pancreas & $\begin{array}{l}\text { Pancreatic exocrine } \\
\text { insufficiency }\end{array}$ & .. & $\begin{array}{l}\text { Cystic fibrosis-related diabetes } \\
\text { mellitus }\end{array}$ & .. \\
\hline Liver & Abnormal liver function & Cirrhosis & Portal hypertension (5-10\%) & Liver transplant \\
\hline Gut & Meconium ileus & .. & $\begin{array}{l}\text { Distal intestinal obstruction } \\
\text { syndrome }\end{array}$ & .. \\
\hline $\begin{array}{l}\text { Reproductive } \\
\text { systems }\end{array}$ & Absence of vas deferens & .. & .. & .. \\
\hline Others & .. & .. & Arthropathy, osteoporosis & .. \\
\hline
\end{tabular}




\section{Clinical Therapeutics for Cystic Fibrosis}

Physiotherapy, also known as physical therapy (PT), can be a treatment for curing symptoms of CF. Since cystic fibrosis causes blocking in airways, doctors perform airways clearance to enable patients' regular breath [13]. Reported by researchers, active cycle of breathing, which is one of airway cleaning technique, has a better long-term effect on pulmonary exacerbation[11]. In order to avoid the outcomes caused by mucus dehydration, clinics often offer airways rehydration to make sure that cilia work properly. The common agents are hypertonic saline (combined with bronchodilator to avoid bronchoconstriction), mannitol, and denufosol. Although physical therapy can solve patients' breathing problems, the undeniable side effects also need to be taken into consideration in the long run.

To deal with inflammations caused by cystic fibrosis, physicians employ antibiotics. Children who get repeated infection by $S$ aureus and $H$ influenzae would receive oral antibiotics to eradicate inflammation. Because cystic fibrosis has major infection on respiratory system, physicians prefer clinical investigation of inhaled tobramycin combined with ultrasonic nebulizer. Effective drug with better technique exhibits considerable improvement on patient's sputum level [14].

In addition to the casual treatment targeting phenotypes, recent developments with effective therapies that correct CFTR function to prevent lung diseases has substantially improved the prospect of disease-modifying treatments and provide a clear objective for future innovation. One of such delivering effective diseasemodifying therapy strategies is using molecular or gene therapy approaches to correct the mutation, whether at DNA or RNA level by bypassing the mutated dysfunction, thus produce a normal functional CFTR protein. Another well-developed approach is the use of small molecules to modulate the CFTR protein and restore functional ion transport [11]. The first small molecular drug that has demonstrated efficacy is ivacaftor. The chemical entity was identified through high-throughput screening and subsequently modified to optimize its therapeutic effect [15]. Preclinical studies have shown that ivacaftor targets most class III mutations, class IV mutations and some other residual function mutations in human and restores mucociliary function. The high-level efficacy has been observed particularly in patients with the Gly551Asp mutation[16]. Efforts have also been made to target patients who are homozygous for the F508del mutation with a combination of a corrector drug to restore trafficking of CFTR and a potentiator to make it functional [17]. In 2019, United State Food and Drug Administration approved the combination use of ivacaftor, with commercial brand name trikafta. This CFTR modulator rectifies body functions that are misled by F508del mutation[18]. According to recent data, around $90 \%$ of patients with F508del mutation have been treated with trikafta. According to the clinical data, trikafta does not have severe side effects on patients. The most common side effects that have been observed are headache, stomach (abdominal) pain, inflamed sinuses, increase in liver enzymes, and increase in a certain blood enzyme creatine phosphokinase. Based on the current technology, trikafta is indeed a promising drug. In general, its effect is also remarkable.

\section{Conclusion}

Threaten by cystic fibrosis, which is caused by gene mutation and can be passed down to next generation, more attention should be paid to solve this public health issue more effectively. We here reviewed cystic fibrosis in the aspects of disease pathogenesis, clinical manifestation as well as clinical therapeutics to gain a more comprehensive understanding of this disease. Appearance of the combination drug trikafta is a promising sign of better dealing with cystic fibrosis. Based on more detailed pathogenesis knowledge and clinical application feedback, more innovative clinical strategies would be within reach.

\section{Reference}

1. Addy, D.P., Cystic fibrosis: current survival and population estimates to the year 2000. Thorax, 1992. 47(4): p. 327.

2. Burgel, P.-R., et al., Future trends in cystic fibrosis demography in 34 European countries. European Respiratory Journal, 2015. 46(1): p. 133.

3. Davis, P.B., Another Beginning for Cystic Fibrosis Therapy. New England Journal of Medicine, 2015. 373(3): p. 274-276.

4. Parkins, M.D., et al., Changing epidemiology and clinical issues arising in an ageing cystic fibrosis population. Therapeutic Advances in Respiratory Disease, 2010. 5(2): p. 105-119.

5. Stoltz, D.A., D.K. Meyerholz, and M.J. Welsh, Origins of Cystic Fibrosis Lung Disease. New England Journal of Medicine, 2015. 372(4): p. 351362 .

6. Henderson, A.G., et al., Cystic fibrosis airway secretions exhibit mucin hyperconcentration and increased osmotic pressure. The Journal of Clinical Investigation, 2014. 124(7): p. 3047-3060.

7. Button, B., et al., A Periciliary Brush Promotes the Lung Health by Separating the Mucus Layer from Airway Epithelia. Science, 2012. 337(6097): p. 937.

8. Boucher, R.C., Bronchiectasis. American Journal of Respiratory and Critical Care Medicine, 2010. 181(10): p. 1017-1019.

9. Ratjen, F.A., Cystic Fibrosis: Pathogenesis and Future Treatment Strategies. Respiratory Care, 2009. 54(5): p. 595.

10. Castellani, C., et al., Consensus on the use and interpretation of cystic fibrosis mutation analysis in clinical practice. Journal of Cystic Fibrosis, 2008. 7(3): p. 179-196.

11. Elborn, J.S., Cystic fibrosis. The Lancet, 2016. 388(10059): p. 2519-2531. 
12. Kelly, T. and J. Buxbaum, Gastrointestinal Manifestations of Cystic Fibrosis. Digestive Diseases and Sciences, 2015. 60(7): p. 1903-1913.

13. Corrigendum. Therapeutic Advances in Chronic Disease, 2016. 7(3): p. 184-184.

14. Richard, B.M., Cystic Fibrosis: Pathogenesis, Pulmonary Infection, and Treatment. Clinical Infectious Diseases, 1995. 21(4): p. 839-849.

15. Yu, H., et al., Ivacaftor potentiation of multiple CFTR channels with gating mutations. Journal of Cystic Fibrosis, 2012. 11(3): p. 237-245.

16. McKone, E.F., et al., Long-term safety and efficacy of ivacaftor in patients with cystic fibrosis who have the Gly551Asp-CFTR mutation: a phase 3, open-label extension study (PERSIST). The Lancet Respiratory Medicine, 2014. 2(11): p. 902-910.

17. Van Goor, F., et al., Correction of the F508del-CFTR protein processing defect in vitro by the investigational drug VX-809. Proceedings of the National Academy of Sciences, 2011. 108(46): p. 18843.

18. Bear, C.E., A Therapy for Most with Cystic Fibrosis. Cell, 2020. 180(2): p. 211. 